\title{
Hypoproliferative human NPC xenografts survived extendedly in the brain of immunocompetent rats
}

Chunhua Liu

Guangzhou Institutes of Biomedicine and Health

Xiaoyun Wang

Guangdong work injury rehabilitation Center

Wenhao Huang

Guangzhou Institutes of Biomedicine and Health

Wei Meng

Guangdong work injury rehabilitation Center

Zhenghui Su

Guangzhou Institutes of Biomedicine and Health

Qi Xing

Guangzhou Institutes of Biomedicine and Health

Heng Shi

City University of Hong Kong

Di Zhang

Guangzhou Institutes of Biomedicine and Health

Min Zhou

Guangzhou Institutes of Biomedicine and Health

Yifan Zhao

Guangzhou Institutes of Biomedicine and Health

Haitao Wang

University of Science and Technology of China

Guangjin Pan

Guangzhou Institutes of Biomedicine and Health

Xiaofen Zhong

Guangzhou Institutes of Biomedicine and Health

Duanqing Pei

Guangzhou Institutes of Biomedicine and Health

Yiping Guo ( $\nabla$ guo_yiping@gibh.ac.cn )

Guangzhou Institutes of Biomedicine and Health https://orcid.org/0000-0003-1628-8312

\section{Research}


Keywords: ESCs, including H1, its gene-modified cell lines for better visualization, HN4

Posted Date: October 23rd, 2020

DOI: https://doi.org/10.21203/rs.3.rs-92873/v1

License: (c) (1) This work is licensed under a Creative Commons Attribution 4.0 International License. Read Full License 


\section{Abstract}

Background: There is a huge controversy about whether xenograft or allograft in the "immune-privileged" brain needs immunosuppression. In animal studies, prevailing sophiscated immunosuppression or immunodeficiency is detrimental for the recipients, which results in short lifespan of animals, and confounds functional behavioral readout of the graft benefit, discouraging long-term follow-up.

Methods: Neuron-restricted human neural progenitor cells (NPCs) were derived from human embryonic stem cells (ESCs, including $\mathrm{H} 1$, its gene-modified cell lines for better visualization, and HN4), propagated for different passages and then transplanted into the brain of immunocompetent rats without use of immunosuppressant. The graft survivals, their cell fates and HLA expression levels were examined over time (up to months after transplantation). We compared the survival capability of NPCs from different passages, and in different transplantation sites (brain parenchyma $\boldsymbol{v s}$. para-and intra-ventricular sites). The host responses to the grafts were also investigated.

Results: Our results show that human ESC-derived neuron-restricted NPCs survive extendedly in adult rat cerebro-parenchyma with no need of immunosuppression whereas a late-onset graft rejection seems inevitable. Both donor HLA expression level and host MHC-II expression level remain relatively low and little change over the long-term survival, and therefore can't predict the late-onset rejection. Human grafts in or close to cerebroventricle are more vulnerable to the immune attack than the intra-striatum grafts. Prevention of graft hyperplasia by using hypoproliferative late-passaged human NPCs further significantly extends the graft survival time. Our new data also shows that a subpopulation of host microglia upregulate MHC-II expression in response to the human graft, but fail to present the human antigen to the host immune system, suggestive of the immune-isolation role of the blood-brain barrier (BBB).

Conclusions: The present study confirms the "immune privilege" of the brain parenchyma, and more importantly, unveils that choosing hypoproliferative NPCs for transplantation can benefit graft outcome in terms of both lower tumor-genic risk and the prolonged survival time without immunosuppression.

\section{Background}

It is now well accepted that the pluripotent stem cell-based cell therapy holds the promise for traditionally incurable neural diseases. Prior to translation into human clinical trials, thorough preclinical in vivo efficacy testing of in vitro induced human neural progenitor cells has to be performed, where rodents and non-human primates are the most important and indispensable animal models. Most xenograft or allograft experiments used high doses of immunosuppressant throughout the lifespan of the animals or use immunodeficiency animals to avoid rejection. However, not surprisingly, overdosed immunosuppression or immunodeficiency results in susceptibility to infections and thus short lifespan of animals, which contrasts to the prolonged time windows required by human neural cells to fully mature and integrate into host brain networks ${ }^{1-3}$. Even more, long-term frequent injection of animals is labor- 
intensive for personnel, painful and stressful for the animals, which has confounded functional behavioral readout of the graft benefit, and discouraged long-term animal studies ${ }^{4}$.

The effort to balance the cell rejection and immunosuppression to reduce the side effects has never stopped. The major route of graft rejection occurs via the recognition of foreign protein molecules (e.g. MHC molecules) by the host immune system. The rate at which allografts are rejected depends on both MHC disparities and transplantation site $^{5}$. Previous studies have reported that human and mouse fetal/embryonic cells including embryonic stem cells and their derivatives, express low level of MHC-I, MHC-II and/or co-stimulatory molecules ${ }^{6-13}$. And the central nervous system is a so called immuneprivileged site where the immune response to allografts is considerably restricted ${ }^{14-16}$. Previous studies have succeeded in transplantation of allogeneic (with animal donor cells) and xenogeneic (with animal or human donor cells) fetal/ embryonic neural cells in animals without immunosuppression ${ }^{17-21}$. More than 20 years of clinical applications of embryonic mesencephalic allografts for the therapy of Parkinson's disease also demonstrated lack of detectable systemic humoral/cellular allogeneic response in human recipients under no immunosuppression condition ${ }^{16,22}$. This, however, has been challenged by some other opposite experimental and clinical evidence ${ }^{23-26}$. A clinical study has reported that 4 in 12 Huntington's disease patients grafted with fetal neural cells demonstrated alloimmunisation and the fifth patient showed overt rejection 14 months after grafting, which is reversible with immunosuppressive treatment ${ }^{26}$. All these debates implied that there is still quite a lack of knowledge on the immunological graft-host interactions in the central nervous system (CNS). A more precise knowledge about survival of human neural stem cells in animal brain without immunosuppression, and the mechanisms underlying the rejection in the CNS, may prevent unnecessarily excessive suppression of immune system in preclinical animal studies, even in patients, warranting the present study.

\section{Materials And Methods}

All experiments were conducted in accordance with the Guide for the Care and Use of Laboratory Animals of the National Institute of Health (Publication No. $80-23$, revised 1996) and were approved by the Experimental Animal Ethics Committee at Guangzhou Institutes of Biomedicine and Health (GIBH), Chinese Academy of Sciences (IACUC NO. 2012008).

\section{Human embryonic stem cell culture and neural induction}

Two human ESCs line, H1 (Passage 60-65, Wicell, Madison, WI, USA) and HN4 (Passage 20-30), were adopted in the present study. All these pluripotent cells were cultured on plates coated with Matrigel (BD Biosciences, San Jose, CA, USA) in mTesR1 medium (Stemcell Technologies, Vancouver, BC, Canada), and routinely passaged by EDTA (Ethylene Diamine Tetraacetic Acid, $0.5 \mathrm{mM}$ ) dissociation every 4-5 days. 
Neural induction was performed as previously reported ${ }^{27,28}$, by dual inhibition of SMAD signaling with empirical modifications to get highly homogenous neural progenitor cells (NPCs) of dorsal forebrain identity. Briefly, hESCs in monolayer culture were first induced to neuroepithelia cells in N2B27 medium (DMEM/F12: Neurobasal 1:1, 0.5\% N2, 1\% B27, 2 mM Glutamax, 1 x NEAA, $5 \mu \mathrm{g} / \mathrm{mL}$ insulin, $2 \mu \mathrm{g} / \mathrm{mL}$ heparin) containing $5 \mu \mathrm{M}$ SB431542 and $5 \mu \mathrm{M}$ Dorsomorphin for 8 days. After passaging, the cells were cultured in N2B27 medium for another 8 days. $20 \mathrm{ng} / \mathrm{mL}$ bFGF was added upon the appearance of rosette-like structures at Day 12-13 for 3-4 days. Then NPCs were harvested by manually picking of rosette-like structures and propagated on Matrigel-coated plate or as suspended neurospheres in N2B27 medium without bFGF and EGF until transplantation. For early passaged NPC transplantation, NPCs within 26-56 days (Passage 2-5) after onset of neural induction were used, and late passaged NPCs were propagated more than 90 days (more than Passage 8) upon neural induction.

\section{Animal surgery and NPC transplantation}

Both male and female Wistar or Sprague-Dawley (SD) rats aged 6-9 weeks were purchased from Charles River (Beijing, China) or Southern Medical University (Guangzhou, China), and normally fed until an age of 8-12 weeks under SPF environment. Upon transplantation, rats were anesthetized via an intraperitoneal injection of 1.0-1.5\% pentobarbital sodium (40-60 mg/kg body weight). Atropine sulphate (Sigma, $0.05 \mathrm{mg} / \mathrm{kg}$, s.c.) was administrated immediately after anesthesia. The subjects were mounted on the stereotaxic device, and then an incision was made in the scalp, a small hole was drilled over the targeting injection site, enabling us to vertically access the striatum or lateral ventricle. After thorough hemostasis, the NPCs were transplanted. Upon transplantation, the NPCs were digested into single cells with Accutase, and suspended in DMEM/F12 $\left(7.5 \times 10^{4} / \mu \mathrm{L}\right) .10-15 \times 10^{4}$ cells in total were microinjected in a single site(for striatum: AP + 1.0, LM3.2,DV5.2 relative to Bregma; for deep in motor cortex: for striatum: $A P+1.2, L M 2.2, D V 3.2$ relative to Bregma). After suture, the rats were then allowed to recover on a temperature-controlled blanket and returned to their home cages with clean beddings. Antibiotics were applied daily in the first week after transplantation. No immunosuppressant was used in the present study unless otherwise stated in control experiments.

\section{Brain slicing and Immunofluorescence staining}

Rats were anesthetized with a lethal dose of pentobarbital sodium ( $200 \mathrm{mg} / \mathrm{kg}$ body weight, i.p.) and perfused transcardially with $0.9 \% \mathrm{NaCl}$ or with $0.1 \mathrm{M}$ PBS followed by cold and fresh $4 \%$ paraformaldehyde (PFA) in $0.1 \mathrm{M}$ phosphate buffer ( $\mathrm{pH}$ 7.4). Brains were quickly collected, postfixed in $4 \%$ PFA overnight, and cryoprotected for $48-72 \mathrm{~h}$ in $30 \%$ sucrose at $4^{\circ} \mathrm{C}$. Brains were sectioned coronally on a cryostat (Leica CM3050S) at $40 \mu \mathrm{m}$ thickness into $0.1 \mathrm{M}$ PBS and processed for immunostaining.

For immunostaining, free-floating sections were washed in PBS 3 times and permeabilized with 1\% Triton $\mathrm{X}-100$ in PBS for $1 \mathrm{~h}$, followed by incubating in blocking buffer (PBS containing 10\% goat or donkey serum and $0.3 \%$ Triton $\mathrm{X}-100$ ) for $1-2 \mathrm{~h}$. Sections were incubated in the primary antibodies diluted in blocking buffer at $4{ }^{\circ} \mathrm{C}$ on shaker overnight, and then in fluorescein-conjugated secondary antibodies and DAPI for $1 \mathrm{~h}$ at room temperature. Three times washing with PBS (15 minutes for each time on shaker) 
followed each antibody incubation. Sections were mounted on glass slide with anti-fluorescence quenching mounting medium for further imaging and analysis. All images were collected on a Zeiss LSM800 confocal microscope and processed with Zen software, ImageJ software (NIH), and Adobe Photoshop CS4 (Adobe Systems, San Jose, CA).

Primary antibodies used in this study include: anti-hNA (Millipore, MAB1281, 1:500), anti-hNA (Millipore, MAB1281A4,1:100), anti-lba-1 (Wako, 019-19741, 1:1000), anti-CD3 (Abcam, ab5690,1:200), anti-DCX (Cell Signaling,1:500), anti-Map2 (Abcam, ab32454, 1:500), anti-GFAP (Dako, Z0334,1:1000), anti-Nestin (Millipore, ABD69, 1:1000), anti-NeuN (Millipore, MAB377,1:500), c-fos (Santa Cruz, sc-52, 1:200), antiOct4 (Santa Cruz, sc-5279, 1:200), anti-Sox17 (R\&D systems, AF1924, 1:400), anti-AFP (GeneTex, GTX84948, 1:200), anti-HLA-ABC (Abcam, ab70328,1:500), anti-HLA-DR (Abcam, ab223907,1:200), antiKi67 (Abcam,ab15580, 1:1000), anti-MHC-II (Abcam,ab23990,1:100). Secondary antibodies include, Goat anti Rat IgG Fc Secondary antibody FITC Conjugate (Invitrogen, TA2505616, 1:1000); Goat anti Rabbit $\operatorname{lgG}(\mathrm{H}+\mathrm{L}$ ) secondary antibody alexa fluor647 (Invitrogen, A32733,1:500); Donkey anti Goat $\lg \mathrm{G}(\mathrm{H}+\mathrm{L}$ ) secondary antibody alexa fluor647 (Invitrogen, A21447,1:500); Goat anti Mouse $\lg G(H+L)$ secondary antibody alexa fluor568 (Invitrogen, A11004,1:500); Goat anti Mouse $\lg \mathrm{G}(\mathrm{H}+\mathrm{L}$ ) secondary antibody alexa fluor488 (Invitrogen, A11001,1:500).

\section{Rabies virus injection}

For retrograde monosynaptic tracing, we used another genetically modified human embryonic stem cell line(H1-CAG-GTRqp) that constitutionally expresses rabies virus glycoprotein, avian TVA receptor(required for selective infection with EnvA-pseudotyped glycoprotein-deleted rabies virus $(\Delta G R V)$ ) and EGFP under control of the human CAG promoter. One week before sacrifice of the animals, rabies virus for retrograde monosynaptic tracing was injected at $0.5 \mathrm{~mm}$-distance above the transplantation site and rats were perfused 7 days later. The titer of the virus was approximately $1.0 \times 10^{7} \mathrm{IU} / \mathrm{ml}$ tested before frozen storage at $-80^{\circ} \mathrm{C}$. A pulled glass micropipette (outer diameter of tip: $30-40 \mu \mathrm{m}$ ) connected to a microinjector (Stoelting, 53311) was used for virus injection. A volume of $1.0 \mu$ lirus was injected in a single site, with a velocity of $0.1 \mu \mathrm{l} / \mathrm{min}$. To reduce backflow after injection, the glass micropipette was left in place for another 10 minutes before slowly withdrawing.

\section{Data analysis}

Quantitative analyses were done by an operator blind to the experiment design. For statistical analysis of the population data, three animal samples from each group were randomly selected to do immunostaining of specific markers. Unpaired t-test was used to examine the differences between two groups. Analysis of variance (ANOVA) followed by Tukey's Multiple Comparison Test for post-hoc analyses was used to examine the differences among three or above groups. For graft survival curve comparison, Log-rank test was used. GraphPad Prism software (v6.0) was used to run the tests. Data were considered significantly different when $p$ was $<0.05$. ${ }^{*} p<0.05 ;{ }^{* \star} p<0.01$.

\section{Results}




\section{Human NPCs survived healthy in the cerebro-parenchyma of adult rats without immunosuppression}

In the first series of experiments, to directly visualize the survival of grafts, we used 2 reporter human cell lines (H1-CAG-DsRed or H1-CAG-GFP) that were previously generated in our lab with constitutive expression of DsRed or GFP reporter gene, at AAVS1 locus of H1 human embryonic stem cell line cells (hESC) through CRISPR/Cas9 gene targeting ${ }^{28}$.

Early passaged (P2-P5, Day 26-56 after neural induction onset) human H1-CAG-DsRed derived NPCs were transplanted into the intact striatum of immunocompetent adult rats. Within 4 weeks after transplantation, grafts survived healthy in ALL animals with no need of immunosuppression, and no obvious immune rejection was observed in any time point, from 4 days(dpt) to 4 weeks (wpt) posttransplantation( (0/22 animals). Human cells were identified by co-localization of human specific nuclear antigen (hNA) and DsRed (Fig. 1A). The first case of massive cell death of human grafts (due to the immuno-rejection, see below) happened at 5 wpt, with a rejection rate of $15.4 \%(2 / 14)$ at $5-6$ wpt. The rejection rate abruptly increased to more than $50 \%(9 / 16)$ at $8-12$ wpt (Fig. 1B-C). Surprisingly, although dead cells (not unequivocally from engrafted cells) might be observed in the injection track or around due to stab injury or secondary inflammation, no brains harbored both live and dead human cell mass within the graft core colony, implicating that the massive graft necrosis was a transient process and took place as an all-or-nothing event.

We repeated the experiment by using another hESC line (HN4, ATCC). All rats (3/3) with intra-striatum HN4 graft had live graft colonies when sacrificed at 4 wpt, whereas overt rejections occurred in 8-wpt rats (4/4), probably due to a high proliferation rate of this batch of HN4 NPCs in a retrospective analysis (Fig.s1).

\section{Engrafted human NPCs had a neuron-restricted cell fate}

We used early passaged NPCs to transplant in most of our experiments. After settled down (usually within 2 wpt), these engrafted human NPCs started to expand, and formed rosette-like structure at 3-5 wpt, which disappeared at longer time points. None of the host brains harbored a teratoma within the observation time window (H\&E staining, Fig.S2A). It is reassuring that immunostaining of the grafts failed to detect any human cells positive for OCT4, AFP and sox17 as markers for residual pluripotent or non-neural cells (Fig.S2B). Notably, all animals lived healthy without any signs of depression or anxiety before sacrifice.

Although quite a few human cells migrated away along white matters tract or blood vessels(Fig.S2C), the majority of the engrafted cells stayed in the injection sites and dispersed within the vicinity, forming a graft colony (Fig. 1A). In the graft colonies, a large number of cells expressed NESTIN (neural stem cell marker), and/or early neuronal markers, like DCX (neuroblast marker), TUJ1 (early neuron precursor) at 3-8 wpt(Fig. 2A), indicative of an early neuronal differentiation stage. Many graft cells also expressed MAP2 (another neuron marker) during this period, while NeuN expression only manifested by 8-12 wpt (Fig. 2A), supporting the previous notion that prolonged time window is needed for human NPCs to 
differentiate into mature neurons. We failed to detect GFAP expression in human cells at any time pointed up to 12 wpt (Fig. 2A), indicative of highly pure neuron-restricted human NPCs transplanted.

We further investigated whether the survived human neurons could well incorporate into host neural circuits without immunosuppression by using retrograde monosynaptic tracing method we recently reported ${ }^{28}$. We used another genetically modified human embryonic stem cell line(H1-CAG-GTRqp) that constitutionally expresses rabies virus glycoprotein, avian TVA receptor(required for selective infection with EnvA-pseudotyped glycoprotein-deleted rabies virus ( $\triangle \mathrm{GRV})$ ) and EGFP under control of the human CAG promoter. At $11 \mathrm{wpt}$, rabies virus $(\triangle \mathrm{GRV})$ was injected at $0.5 \mathrm{~mm}$-distance above the transplantation site (deep in motor cortex) and rats were perfused 7 days later. The infected human cells co-expressed EGFP and mCherry fluorescence (as the starter neurons, EGFP + mCherry+, Fig. 2B-a), which received the afferent projections from the host neurons that only expressed mCherry (as the traced neurons, EGFPmCherry+) in both the ipsilateral cortex (Fig. 2B-b) and the ipsilateral thalamus (Fig. 2B-c).

All these data showed that the grafted NPCs were highly neuronal-restricted, could properly migrate, differentiate into neurons as expected, further mature and incorporate into host neural network under no immunosuppression settings.

\section{Late-onset cell death was due to immune rejection}

As mention above, at late time points (i.e. 5, 6, 8, $12 \mathrm{wpt)}$ ), we only saw massive dead human cells deposited in some animals, as identified by dusty DsRed fluorescent signals without cell morphology, where cavitation and cracks were sometimes conspicuous (Fig.S3A and Fig. S3B). The hNA positive immunoreactivity was also found desperately messy and weak, indicative of rapid loss of cellular components of engrafted cells (Fig. 1B). Instead, the graft colony was reoccupied by host cells, many of which with irregular cell body showed robust Iba1 expression, a marker of microglia or monocytes, implicating undergoing destructive phagocytosis of the dead graft cells by host (Fig. 3A).

To figure out whether this seemingly spontaneous late-onset cell death was due to adaptive immunorejection, lack of oxygen and glucose supply or other reasons, e.g. spontaneous apoptosis, we further did H\&E staining, and CD3 immunostaining, a T-lymphocyte marker. In H\&E staining, the rejected grafts were characterized with dense and clustered leucocyte infiltration (Fig.S3B), and many of them were CD3 positive with lymphocyte morphology (Fig. 3B). Perivascular lymphocytic cuffing was present within the graft and at the graft-host interface. We also detected heavy host IgG deposition within rejected graft, suggestive of the precipitation of humoral immunity (Fig. 3C). Moreover, the intense infiltration of host inflammatory cells (microglia and T-lymphocytes) and IgG deposition was circumscribed to necrotic graft remnants, and largely spared the neighboring host structures. In contrast, non-rejected human grafts were completely devoid of any leukocyte infiltrate and IgG deposition, except weak signals detected along the injection track at early time points (Fig. 3B-C).

In another experiment, we transplanted human NPCs into two different parenchymal sites of the same rats with an interval distance of $7 \mathrm{~mm}(\mathrm{n}=12)$, and examined at 5 wpt. Human grafts were found dead at 
both sites in 4 recipients, while others only contained live human graft colony (Fig.S4). This "Both-orNone" phenomenon ruled out the possibility that the elimination of human cells was mainly due to lack of oxygen and glucose supply or spontaneous apoptosis. The reasonable explanation for this is that hosts sensitized by either graft would launch immune attack on both.

Taken together, our results suggested that immuno-rejection might be the major contributing factor to the late-onset death of human grafts. This conclusion also borrowed supports from our another experiment showing that human grafts survived in rat brain up to 6 months when daily cyclosporine immunosuppression was used ${ }^{28}$.

\section{HLA-ABC expression was NOT the trigger for the late-onset rejection of human NPCs}

Studies have demonstrated that $\mathrm{MHC}$ expression are of prime importance in allograft rejection and may be the precedent step to xenograft rejection ${ }^{29,30}$. Human breast cancer cells (MDA-MB-231), which express high-level HLA-ABC, were quickly rejected after transplanted into the striatum (Fig.S5A). Consistent with previous studies from other labs, human ESC-derived NPCs displayed a low HLA-ABC expression level (Fig.S5B), which might protect them from immune attack at the time of graft when the blood-brain barrier (BBB) was transiently disturbed. So the next question is whether engrafted human NPCs would upregulate the HLA-ABC expression during the survival time and then trigger the late-onset cell rejection. The immunostaining results showed that HLA-ABC expression remained barely detectable in all non-rejecting brains at any time point up to 12 wpt (Fig.S5C), and very few HLA-ABC positive cells could be occasionally identified if given much prolonged survival time(data from another experiment with immune-suppression). We also failed to detect HLA-DR expression (Fig.S5C), which has long been strongly associated to transplant rejection ${ }^{31}$. Thus, the expressions of HLA-ABC and HLA-DR antigens retained a low level in vivo during our observation time window, and should not be responsible for initiation of the late- onset human cell rejection.

\section{Intra-cerebroventricular human NPC grafts were vulnerable to immune attack more than the intra-striatum grafts}

Notably, in some rejecting cases, we noticed that the grafts protruded into the paraventricular area, where human cell antigen might more easily get access to the immune system and trigger the immune reaction. Recent findings have revealed a role for circumventricular organs, such as the choroid plexus, as gateways in the trafficking of peripheral leukocytes to the CNS ${ }^{32,33}$. The meningeal lymphatic vessel was found to drain cerebrospinal fluid directly into cervical lymph nodes ${ }^{34}$. So, we hypothesized that the rupture of the paraventricular structure should be detrimental for survival of human grafts. If this is true, the intra-cerebroventricular human grafts should not be able to survive long in immunocompetent rats. So we transplanted human NPCs into the lateral ventricles to see whether the brain ventricles are as immuneprivileged as parenchyma. Four weeks later, we did not see any live graft in these animals $(n=6)$. The ventricles were not enlarged, and only trace of dead cells sparsely scattered in the ventricles, indicative of quick depletion of human cells. Given that the ventricle of rat and mouse brain has been shown to be a 
favorable site for neural grafts in immunodeficient xenogeneic hosts ${ }^{35}$, the death of intracerebroventricular graft was unlikely due to non-immunologically environmental factors. More importantly, intra-cerebroventricular graft also induced death of human NPCs deposited on the upper and lower banks of the ipsilateral lateral ventricles or in the fimbria of the hippocampus with T-lymphocyte infiltration. In another experiment, human NPCs were injected close to the lateral ventricle (within $0.5 \mathrm{~mm}$ to the lateral ependymal wall), and no human grafts survived at 6 wpt $(n=5)$. All these results suggested that the rupture of the paraventricular structure was detrimental for the graft survival in immunocompetent rats (the rejection rate: $12.5 \%$ (intra-cerebro-parenchyma, $1 / 8$ at $6 \mathrm{wpt}$ ) vs. $100.0 \%$ (intra-/ para-cerebroventricular, 11/11).

\section{Hypoproliferative human NPC grafts further extended their survival}

Low immunogenicity of human NPC grafts (with low HLA-ABC and HLA-DR expression level) could protect them from the host immune attack. Another arm of the CNS immune-privilege is the BBB, which protects the CNS from the immune attack to a great extent ${ }^{5}$. The late-onset human graft rejection could be also due to the compromise of this barrier. As mentioned above, the early passaged human NPCs started significant expansion after settled down in the rat brain. When these grafts grew too quickly to disperse promptly, they easily formed a large and incongruous hyperplastic core within short time (Fig. 1 and other figures), which might impose a continuous compression on the surrounding tissues and destruct the local vascular system or break into the paraventricular organ, resulting in intermittent perturbations of local BBB or ventricle-meningeal lymphatic system and finally graft rejection as described above. The heavy T-lymphocyte infiltration and IgG deposition within the rejected graft did demonstrate such vascular or paraventricular damages. So we hypothesized that the rapid and sustained enlargement of grafts might account for the human cell rejection in our experiments. We turned to late passaged NPCs (beyond 90 days after neural induction onset), which exhibited a much lower proliferation capability (Fig. 4A-C). Even better than expected, up to our longest survival time (more than 4 $\mathrm{mpt})$, the late human NPCs survived in the overwhelming majority of animals (2/2 at 4-5w, 9/10 at $8 \mathrm{wpt}$, $5 / 6$ at $12 \mathrm{wpt}, 3 / 4$ at more than $4 \mathrm{mpt}$ ) without massive cell death. Graft survival analysis showed that late passaged NPCs with low proliferation survived significantly longer than early passaged NPCs (Fig. 4D, Log-rank test, $P<0.01^{\star \star}$ ). The late hypoproliferative NPC grafts formed a much smaller graft core than the early passaged graft at $4 \mathrm{wpt}$ and thereafter (Fig. 4A vs. B). Moreover, the long-term survived human grafts migrated along the white matters into a wide territory of ipsilateral cerebral cortex without a conspicuous graft core (FigS6).

However, further studies are needed to demonstrate that such a rupture of BBB or paraventricular structure occurs prior to the cell rejection, and to determine what antigens on the human cells would trigger this immune reaction in that the expression level of HLA-ABC and HLA-DR antigen remained barely detectable.

\section{The host microglia responded moderately to human NPC grafts}


Previous studies have suggested important roles played by microglia in both the antigen presenting and immune attack phases of intracerebral allograft rejection ${ }^{36,37}$. In the absence of CNS inflammation, microglia showed resting ramified morphology typically of non-overlapping long branching processes and a small cellular body (Fig.S7A). One of the characteristics of microglia in vivo is their ability to survey the parenchyma from their static tiling position ${ }^{38}$ and to react quickly to even small pathological changes. In response to the human grafts, the host microglia proliferated, migrated into and recolonized the graft area, where host cells including microglia had been squeezed away by the enlarged human graft (Fig. 5). Although individual phagocytic microglia were occasionally observed within the graft, most of the invading microglia showed a migrating rod-like or "cup" shape, or mildly activated with couple thick processes (Fig. 5). This quite differed from their resting ramified morphology in the intact host brain region (Fig.S7A), and also sharply contrasted to the massive end-stage phagocytic microglia in the rejecting region, which showed intensive activation typically with hypertrophic cell body, ring or irregular shape and severely retracted branches (Fig. 3B, Fig.S7B).

We further examined the host MHC-II expression in the non-rejecting animals to seek any early cues underlying the late-onset immuno-rejection. We failed to find any MHC-Il expression in astrocytes and endothelial cells (or below the detectable level of the present method) in brain parenchyma, but a subpopulation of microglia within healthy graft area upregulated MHC-Il expression (Fig. 5A). Nearly all MHC-Il positive microglia were mildly activated except individuals with phagocytic profiling, while resting microglia never showed any MHC-II expression. Notably, the host MHC-II expression level remained relatively low and showed no significant increase over the long-term surviving period (Fig. 5C), implying that the MHC-II positive microglia failed to present antigens to the host immune system and trigger downstream responses, suggestive of an immune-isolation role by the intact BBB and ependymal wall and the "immune privilege" of CNS. Similarly, as mentioned below, although lipopolysaccharide (LPS) challenge increased MHC-Il expression within the graft (Fig. 5B), this did not significantly increase the rejection rate of human cells. LPS also induced a sparse MHC-Il upregulation in the microglia in a brainwide manner, indicative of undergoing inflammation (Fig.S8).

All these results proposed that the mild elevation of $\mathrm{MHC}$-II expression in the microglia in response to live human grafts was a sign of exogenous graft-induced inflammation (and/or just small environment changes) or the initial immuno-recognition without downstream immune reaction. The chronological quantification of MHC-II expression showed no gradual signs indicating initiation of rejection in all nonrejecting rats, again supporting the notion that the immuno-rejection was an unexpected and sudden event.

\section{LPS-induced peripheral inflammations did not increase the rejection of human NPC grafts}

To test whether or not peripheral inflammation would induce the rejection of the stable human NPC grafts, we used a well-established LPS challenge paradigm ${ }^{39}$. Rats were injected intraperitoneally with $2.0 \mathrm{mg} / \mathrm{kg}$ LPS for 2 consecutive days at $4.5 \mathrm{wpt}$ when human grafts had settled down. LPS injections induced a brief sickness (e.g. fever, loss of body weight, anorexia and hypokinesia) that lasted for about 3 
days except the body weight changes. At 6 wpt (10 days after the second LPS injection), trace of microglia activation could still be discerned from the lba1 expression level and profiling in a brain-wide manner (Fig.S8). LPS also further increased phagocytic microglia within the graft colony, in circle or ring shape with apparent MHC-II expression (Fig. 5B). Nevertheless, this LPS-induced peripheral inflammation and microglia activation in CNS did not significantly increase the rejection rate of human cells $(1 / 5$ in LPS-challenged VS. 1/6 in non-LPS). In consistent with this, we also did not see upregulated lymphocyte infiltration in the LPS-challenged non-rejecting brains.

\section{Discussion}

In general, our present results confirmed the "immune privilege" of the brain parenchyma behind the BBB and ependymal wall that could substantially protect human ESC-derived NPC xenograft in the brain from rejection, much more reliably than expected. Specifically, human ESC-derived NPCs survived extendedly in adult rat brain parenchyma without immunosuppression, but not in and around the cerebroventricles. And, hypoproliferative late-passaged human NPC grafts further extended their survival up to our longest observation time window(more than 4 months), most likely via lowering the damage risks to the integrity of the BBB and ependymal wall.

Neonatal rodents have long been proven to be ideal recipients for allogenic neural grafts in the CNS with on need of immunosuppression, where the majority of allogenic grafts (globally $80 \%, 40,41$ ) could survive up to several months to even one year. This has been ascribed to the consensus that the injectioninduced BBB damage has restored before the immunological competence established 2 to 3 weeks after birth. Long term allogenic grafts were also reported in adults with transient immunosuppression

exceeding the time necessary for the blood-brain barrier to reform ${ }^{42-44}$, or even without immunosuppression ${ }^{18,45}$. All these results suggested a critical role of BBB intact in prevention of graft rejection. However, now most studies have been using life-long immunosuppression or immunodeficiency animals, showing that CNS immune privilege is not absolute and immunosuppression is necessary.

\section{Prevention of graft hyperplasia would benefit long-term survival of the allogenic NPC grafts}

Whether the transplants rejected or not depends on both the graft immunogenicity and the host immunological status. Many variables contribute to these two factors. For example, cells from different sources (e.g. fetal neural cells vs. embryonic stem cell-derived NPCs, primary vs. after long-term culture 20 may have distinct cellular behaviors, MHC expression patterns and levels after transplantation. The animal disease models have a higher sensitivity to the exogenous cells than intact brains, and different models may bring out the variety in time needed to restore the BBB intact and inflammation level. It has also been reported that grafts in single-cell suspension may permit extensive cell migration, and antibodies may be more destructive to solid pieces of tissue than cell suspension ${ }^{15}$. Therefore, it is really 
difficult to find out where these seemly contradictory conclusions among different studies come from by comparing so many variables side by side, especially some of the details unavailable in the publications.

Nevertheless, it will be much easier to tell, in retrospect, how allogenic grafts behave under no or transient immunosuppression conditions, to avoid rejection from the published data. Notably, the long-term grafts in the neonatal brain always integrated substantially with host neural system, where large hyperplastic core seldom reported, thus remaining un-recognized or un-attacked by the host immunity system outside BBB, whereas the graft hyperplasia was common in injection site of the adult recipients ${ }^{1,28}$. In light of the present data, the most important reason accounting for the long-term xenograft in neonatal brain, we would suggest, might be that the perinatal developmental niche provides an ideal environment for the neural graft to better migrate, differentiate and incorporate. In this study, early passaged human NPCs proliferated significantly and greatly outnumbered the initially engrafted cells. These cells showed limited dispersion and deposited as large hyperplasia in the injection site 8-12 weeks after grafted, which triggered the late-onset cell rejection, possibly via continuously compressing host brain and breaking down the local BBB or ventricle-meningeal lymphatic system. The rejection was largely relieved in late passaged NPC transplants, where only limited cell depositions or small cell clusters were observed at 4 wpt and thereafter. And the long-term surviving human grafts showed wide migration into the host brain territory without conspicuous graft core. Björklund previously hypothesized that extensive cell migration may be the key to the survival of dopaminergic xenografts in adult PD rats ${ }^{18}$. Daniloff and colleagues also demonstrated that a permanent incorporation of the transplanted cells into the host would be important for long-term survival of xenograft in adult rats of denervated hippocampus ${ }^{19}$. In both of these studies, the bulk of the mouse graft in the rat recipient disappeared or was resorbed, and the surviving neurons after 6 months and 17 weeks, respectively, had migrated away from the injection site, with dense innervation to deafferented host targets. Lund et al. also reported that smaller transplants showed significantly less lymphocytic infiltration than large ones ${ }^{40}$. All these results implied that the potential to form hyperplastic foci is detrimental to the graft survival.

Several factors might contribute to the potential to form hyperplastic foci. First, the transplants differentiate according to a timetable dictated by the donor species rather than by the host. In human brain development, cell proliferation occurs during the whole 40 weeks of gestation. Human NPCs require a prolonged time window to fully mature and integrate, thus resulting in higher tendency to overproliferation, which might account for why human fetal cells are more prone to rejection. Second, transplantation in corpus callosum and denervated brain region may benefit the graft dispersion or migration. Wennersten showed human fetal neural cells could survive in contused rat brain 6 months after termination of immunosuppression, scattered in hippocampus and neocortex ${ }^{42}$. Smith et al. also reported long-term survival of human cells (14weeks) in MCAO rats ${ }^{44}$. In both studies, short-term immunosuppression was used until BBB restored.

\section{The role of microglia in graft rejection}


The presence of BBB and the lack (or low level) of cells within the brain expressing major histocompatibility complex (MHC) antigens had been thought to play key role in maintaining CNS immune privilege. However, it has become increasingly apparent that neither factor is absolute. Increased $\mathrm{MHC}$ antigen expression has been demonstrated in brain injury and diseases and as well as in response to neural allograft $23,35,37,46,47$. Several studies ${ }^{36,47}$ have proposed microglia as the APC candidate in CNS by showing a correlation between high MHC-Il expression level in microglia and graft rejection, other than, however showing a convincing cause-result relationship between them ${ }^{46}$. Lawrence etc. reported that microglia were involved in both the immune presenting and immune attack phases of intracerebral allograft rejection ${ }^{36}$, showing that MHC-II gradually upregulated in microglia, but not in astrocytes, in response to the allograft of embryonic hippocampal primordia. We also showed that the MHC-II expression was restricted to microglia. However, unlike the former study, MHC-II expression in our neuronogenic NPC transplantation settings only elevated in a subpopulation of microglia, and retained low with little change over the long-term survival, indicating that $\mathrm{MHC}$-II positive microglia failed to present the donor antigen to the host immune system, due to the immune-isolation of the intact BBB and ependymal architecture. This discrepancy might come from different cell components of the grafts we used. Hippocampal primordia graft consisted of a variety of cell types, including vascular endothelial cells and glial cells, which acquired MHC-I and MHC-II immunoreactivity shortly in the damaged xenogeneic environment and boosted the MHC-II expression of host counterparts ${ }^{36}$. In contrast, our neuronogenic NPC graft sustained a low level of HLA-ABC and HLA-DR antigens, and the host MHC-II expression only upregulated in a subpopulation of microglia, which is more likely indicative of an undergoing graft-induced low-level and tolerable inflammation. Still, Lawrence and colleagues could overclaim the antigen presenting role of microglia, as mentioned by themselves that MHC-II expression in the microglia were less intense than that in the endothelial cells or perivascular cuff, and the initial reactive difference between the allogenic and syngeneic grafts was related to the blood vessels. The donor endothelial cells expressing both MHC-I and MHC-II, might present their own alloantigen directly to circulating host cells after incorporated into the local vasculature. The strong phagocytosis executed by microglia and/or monocytes in the rejecting brains could also be result of rejection rather than the cause, that is, microglia function herein as a housekeeper to cleaning up damaged cells and cellular debris rather than as a killer even within the graft. Therefore, it would be of great interest to further investigate whether deletion of microglia could largely extend the survival of allogenic grafts in our experiment.

\section{Implications for Future Preclinical Studies and Clinical Translations}

Xenograft models are very important for basic research and preclinical studies using human cells for transplantation. Although many studies have shown that the absence of immunosuppression was detrimental to the xenograft of human cells, our new data demonstrated that human ESC-derived NPCs can virtually survive extendedly in the brain of immunocompetent adult rat (up to more than 4 months post-transplantation). More importantly, human NPCs differentiate, migrate, mature and incorporate into host neural network properly, and all animals live healthy without any signs of depression or anxiety 
before sacrifice. In contrast, overdosed immunosuppression or immunodeficiency are always associated with side effects (e.g. resistant to infection, nephropathy ${ }^{48}$, hepatotoxicity ${ }^{49}$, and gingival overgrowth ${ }^{50}$, results in short lifespan of animals, impose stress on animals, confound functional behavioral readout of the graft benefit, discouraging long-term animal studies.

Our present results suggested that Wistar and SD rats, when used as the cerebro-parenchymal xenograft recipients could be took as immune-deficient animals, with no need for immunosuppression. Human NPC grafts either of a limited proliferation capability, or deposited faraway from ventricle-meningeal system (e.g. injected in deep or more lateral brain structures) could achieve longer survival time. Although we only tested human ESC-derived NPCs, this result might be further generalized to donor cells of different species or from different sources ${ }^{51}$. The prerequisite includes a low MHC expression level of the donor cells and a low inflammation level when transplanted. Otherwise, such as in brain injury, either transient immunosuppression exceeding the time needed for BBB restoration should be considered $42,44,52$, or administration of anti-inflammation reagent, such as NSAIDs, would be sufficient to avoid rejection ${ }^{53}$.

Furthermore, peripheral inflammation would not induce the rejection of the stable human NPC grafts, even when the CNS microglia are activated with enhanced MHC-II expression. Our present data provides a good explanation for the clinical evidence that more than 20 years of cellular transplant experience in humans and autopsy findings of Parkinson's disease patients with allogenic fetal neural grafts showed little evidence of immunological reactions without immune suppression.

\section{Conclusions}

Our data proved that the "immune privilege" of central nervous system could substantially protect human ESC-derived NPC xenograft from rejection in the rat brains. This CNS graft tolerance is not due to the antihuman specific $\mathrm{T}$ cell clonal deletion ${ }^{54}$, but largely to the immune-isolation behind the BBB and ependymal wall. The late-onset graft rejection could be ascribed to the sudden and intermittent disruption of BBB or ventricle-meningeal lymphatic systems without remarkable and gradual buildup of donor and host MHC levels and inflammation level prior to the rejection. Choosing hypoproliferative NPCs for transplantation can benefit graft outcome in terms of both lower tumor-genic risk and the prolonged survival time without immunosuppression.

However, when coming to clinical translation, our current study shows limitations, including the relatively short observation time window post transplantation and only rats used. Accordingly, large-animal models and a prolonged postgrafting survival beyond half a year or even more than 1 year will be needed to confirm a permanent human ESC-NPC engraftment in xenogeneic settings.

\section{Abbreviations}

ESC

embryonic stem cell; 
NPC

neural progenitor cell;

LPS

lipopolysaccharide;

BBB

blood-brain barrier;

$\mathrm{MHC}$

major histocompatibility complex;

CNS

central nervous system ;

HLA

human leukocyte antigen

$H \& E$

Hematoxylin and eosin

\section{Declarations}

\section{Availability of data and materials}

All the data supporting the conclusions of this article is included within the article and the supplemental materials.

\section{Acknowledgements:}

We thank Dr. Juan Du and Zhiwu Jiang from the GIBH, CAS for commenting on this manuscript, and Dr. Shengbiao Li for technical assistance and materials with the immunostaining of endodermal markers.

\section{Funding:}

This work was partially supported by the Chinese Ministry of Science and Technology (2017YFA0103101), the National Natural Science Foundation of China (81771356, U1601228); the Science and Technology Programs of Guangzhou (201803040016, 201804020052); the Key Research \& Development Program of Guangzhou Regenerative Medicine and Health Guangdong Laboratory(2018GZR110104003); the Frontier and Key Technology Innovation Special Grant from the Department of Science and Technology of Guangdong Province (2014B020225006, 2015B020228003, 2020B1212060052).

\section{Author information}

Author Contributions: C.L. contributed to the experiments of cell transplantation, neural induction and NPC maintaining, data analysis, and figure construction. X.W did the immunostaining, data analysis, and manuscript writing. W.M. and D.Z. contributed to the animal surgery, the postoperative handling and animal perfusion. Z.S., W.H. and M.Z. performed the neural induction. H.S. did the postoperative handling 
and animal perfusion. Q.X. contributed to the animal surgery and human ESC line construction. H.W. contributed to experiment design. Y.Z. participated in double-blind experiment. G.P. supervised the experiments. X.Z. contributed to experiment design, data analysis and interpretation. D. P. conceived the experiments and supervised the experiments. Y.G. contributed to conception and design, data analysis and interpretation, and manuscript writing. All authors approved the submitted version.

\section{Corresponding authors}

Correspondence to Yiping Guo (guo_yiping@gibh.ac.cn), Xiaofen Zhong (zhong_xiaofen@gibh.ac.cn) and Duanqing Pei (pei_duanqing@gibh.ac.cn).

\section{Ethics declarations}

\section{Ethics approval and consent to participate}

All experiments were conducted in accordance with the Guide for the Care and Use of Laboratory Animals of the National Institute of Health (Publication No. 80-23, revised 1996) and were approved by the Experimental Animal Ethics Committee at Guangzhou Institutes of Biomedicine and Health (GIBH), Chinese Academy of Sciences (IACUC NO. 2012008). All efforts were conducted to minimize animal number and suffering.

\section{Consent for publication}

Not applicable.

\section{Competing interests}

The authors have no financial disclosures or conflict of interest with the research presented here.

\section{References}

1. Real R, et al In vivo modeling of human neuron dynamics and Down syndrome. Science 362(2018).

2. Lu P, et al. Prolonged human neural stem cell maturation supports recovery in injured rodent CNS. J Clin Invest. 2017;127:3287-99.

3. Nicholas $\mathrm{CR}$, et al. Functional maturation of hPSC-derived forebrain interneurons requires an extended timeline and mimics human neural development. Cell Stem Cell. 2013;12:573-86.

4. Jensen MB, Krishnaney-Davison R, Cohen LK, Zhang SC. Injected Versus Oral Cyclosporine for Human Neural Progenitor Grafting in Rats. J Stem Cell Res Ther Suppl. 2012;10:003.

5. Drukker M. Immunogenicity of human embryonic stem cells: can we achieve tolerance? Springer Semin Immunopathol. 2004;26:201-13.

6. Drukker $\mathrm{M}$, et al. Human embryonic stem cells and their differentiated derivatives are less susceptible to immune rejection than adult cells. Stem Cells. 2006;24:221-9. 
7. Odeberg J, Piao JH, Samuelsson EB, Falci S, Akesson E. Low immunogenicity of in vitro-expanded human neural cells despite high MHC expression. J Neuroimmunol. 2005;161:1-11.

8. Itakura G, et al. Low immunogenicity of mouse induced pluripotent stem cell-derived neural stem/progenitor cells. Sci Rep. 2017;7:12996.

9. Chen HF, et al. Characteristic expression of major histocompatibility complex and immune privilege genes in human pluripotent stem cells and their derivatives. Cell Transplant. 2015;24:845-64.

10. Drukker $\mathrm{M}$, et al. Characterization of the expression of $\mathrm{MHC}$ proteins in human embryonic stem cells. Proc Natl Acad Sci U S A. 2002;99:9864-9.

11. Hori J, et al. Neural progenitor cells lack immunogenicity and resist destruction as allografts. Stem Cells. 2003;21:405-16.

12. Mammolenti M, Gajavelli S, Tsoulfas P, Levy R. Absence of major histocompatibility complex class I on neural stem cells does not permit natural killer cell killing and prevents recognition by alloreactive cytotoxic T lymphocytes in vitro. Stem Cells. 2004;22:1101-10.

13. Magliocca JF, Held IK, Odorico JS. Undifferentiated murine embryonic stem cells cannot induce portal tolerance but may possess immune privilege secondary to reduced major histocompatibility complex antigen expression. Stem Cells Dev. 2006;15:707-17.

14. Ferguson TA, Green DR, Griffith TS. Cell death and immune privilege. Int Rev Immunol. 2002;21:15372.

15. Barker CF, Billingham RE. Immunologically privileged sites. Adv Immunol. 1977;25:1-54.

16. Barker RA, Widner H. Immune problems in central nervous system cell therapy. NeuroRx. 2004;1:47281.

17. Strnadel J, et al Survival of syngeneic and allogeneic iPSC-derived neural precursors after spinal grafting in minipigs. Sci Transl Med 10(2018).

18. Bjorklund A, Stenevi U, Dunnett SB, Gage FH. Cross-species neural grafting in a rat model of Parkinson's disease. Nature. 1982;298:652-4.

19. Daniloff JK, Low WC, Bodony RP, Wells J. Cross-species neural transplants of embryonic septal nuclei to the hippocampal formation of adult rats. Exp Brain Res. 1985;59:73-82.

20. Armstrong RJ, et al. Porcine neural xenografts in the immunocompetent rat: immune response following grafting of expanded neural precursor cells. Neuroscience. 2001;106:201-16.

21. Englund U, Fricker-Gates RA, Lundberg C, Bjorklund A, Wictorin K. Transplantation of human neural progenitor cells into the neonatal rat brain: extensive migration and differentiation with long-distance axonal projections. Exp Neurol. 2002;173:1-21.

22. Freed CR, et al. Transplantation of embryonic dopamine neurons for severe Parkinson's disease. $\mathrm{N}$ Engl J Med. 2001;344:710-9.

23. Nicholas MK, Antel JP, Stefansson K, Arnason BG. Rejection of fetal neocortical neural transplants by H-2 incompatible mice. J Immunol. 1987;139:2275-83. 
24. Lindvall 0 , et al. Grafts of fetal dopamine neurons survive and improve motor function in Parkinson's disease. Science. 1990;247:574-7.

25. Warfvinge $\mathrm{K}$, et al. Xenotransplantation of human neural progenitor cells to the subretinal space of nonimmunosuppressed pigs. J Transplant 2011, 948740 (2011).

26. Krystkowiak $P$, et al. Alloimmunisation to donor antigens and immune rejection following foetal neural grafts to the brain in patients with Huntington's disease. PLoS One. 2007;2:e166.

27. Wang $\mathrm{H}$, et al. 5-HT2 receptors mediate functional modulation of GABAa receptors and inhibitory synaptic transmissions in human iPS-derived neurons. Sci Rep. 2016;6:20033.

28. Xing Q, et al. Retrograde monosynaptic tracing through an engineered human embryonic stem cell line reveals synaptic inputs from host neurons to grafted cells. Cell Regen (Lond). 2019;8:1-8.

29. Pakzaban P, Deacon TW, Burns LH, Dinsmore J, Isacson O. A novel mode of immunoprotection of neural xenotransplants: masking of donor major histocompatibility complex class I enhances transplant survival in the central nervous system. Neuroscience. 1995;65:983-96.

30. Petrus-Reurer S, et al. Generation of Retinal Pigment Epithelial Cells Derived from Human Embryonic Stem Cells Lacking Human Leukocyte Antigen Class I and II. Stem Cell Reports. 2020;14:648-62.

31. Dreyer GJ, de Fijter JW. Transplanting the Elderly: Mandatory Age- and Minimal Histocompatibility Matching. Front Immunol. 2020;11:359.

32. Baruch K, et al. Aging. Aging-induced type I interferon response at the choroid plexus negatively affects brain function. Science. 2014;346:89-93.

33. Ransohoff RM, Kivisakk P, Kidd G. Three or more routes for leukocyte migration into the central nervous system. Nat Rev Immunol. 2003;3:569-81.

34. Absinta $\mathrm{M}$, et al Human and nonhuman primate meninges harbor lymphatic vessels that can be visualized noninvasively by MRI. Elife 6(2017).

35. Mason DW, et al. The fate of allogeneic and xenogeneic neuronal tissue transplanted into the third ventricle of rodents. Neuroscience. 1986;19:685-94.

36. Lawrence JM, Morris RJ, Wilson DJ, Raisman G. Mechanisms of allograft rejection in the rat brain. Neuroscience. 1990;37:431-62.

37. Poltorak M, Freed WJ. Immunological reactions induced by intracerebral transplantation: evidence that host microglia but not astroglia are the antigen-presenting cells. Exp Neurol. 1989;103:222-33.

38. Huang $Y$, et al. Repopulated microglia are solely derived from the proliferation of residual microglia after acute depletion. Nat Neurosci. 2018;21:530-40.

39. Chen Z, et al. Lipopolysaccharide-induced microglial activation and neuroprotection against experimental brain injury is independent of hematogenous TLR4. J Neurosci. 2012;32:11706-15.

40. Lund RD, Rao K, Hankin MH, Kunz HW, Gill TJ. 3rd. Transplantation of retina and visual cortex to rat brains of different ages. Maturation, connection patterns, and immunological consequences. Ann $\mathrm{N}$ Y Acad Sci 495, 227-41 (1987). 
41. Lund RD, Rao K, Kunz HW, Gill TJ. 3rd. Instability of neural xenografts placed in neonatal rat brains. Transplantation. 1988;46:216-23.

42. Wennersten A, et al. Sustained survival of xenografted human neural stem/progenitor cells in experimental brain trauma despite discontinuation of immunosuppression. Exp Neurol. 2006;199:339-47.

43. Rota Nodari L, et al. Long-term survival of human neural stem cells in the ischemic rat brain upon transient immunosuppression. PLoS One. 2010;5:e14035.

44. Smith EJ, et al. Implantation site and lesion topology determine efficacy of a human neural stem cell line in a rat model of chronic stroke. Stem Cells. 2012;30:785-96.

45. Lee SR, et al. Long-term survival and differentiation of human neural stem cells in nonhuman primate brain with no immunosuppression. Cell Transplant. 2015;24:191-201.

46. Shinoda $M$, et al. Microglial cell responses to fetal ventral mesencephalic tissue grafting and to active and adoptive immunizations. Exp Neurol. 1996;141:173-80.

47. Pollack IF, Lund RD, Rao K. MHC antigen expression and cellular response in spontaneous and induced rejection of intracerebral neural xenografts in neonatal rats. Restor Neurol Neurosci. 1990;1:347-60.

48. Chander V, Chopra K. Effect of molsidomine and L-arginine in cyclosporine nephrotoxicity: role of nitric oxide. Toxicology. 2005;207:463-74.

49. Laupacis A, Keown PA, Ulan RA, Sinclair NR, Stiller CR. Hyperbilirubinaemia and cyclosporin A levels. Lancet. 1981;2:1426-7.

50. Guo J, Wang W, Yao L, Yan F. Local inflammation exacerbates cyclosporine a-induced gingival overgrowth in rats. Inflammation. 2008;31:399-407.

51. Ortega JD, Sagen J, Pappas GD. Short-term immunosuppression enhances long-term survival of bovine chromaffin cell xenografts in rat CNS. Cell Transplant. 1992;1:33-41.

52. Zhang G, et al. Stable Intracerebral Transplantation of Neural Stem Cells for the Treatment of Paralysis Due to Ischemic Stroke. Stem Cells TransI Med. 2019;8:999-1007.

53. Chen Z, et al. MHC mismatch inhibits neurogenesis and neuron maturation in stem cell allografts. PLoS One. 2011;6:e14787.

54. Starr TK, Jameson SC, Hogquist KA. Positive and negative selection of T cells. Annu Rev Immunol. 2003;21:139-76.

\section{Supplementary Information}

Supplementary information is available at Stem Cell Research \& Therapys website

\section{Figures}




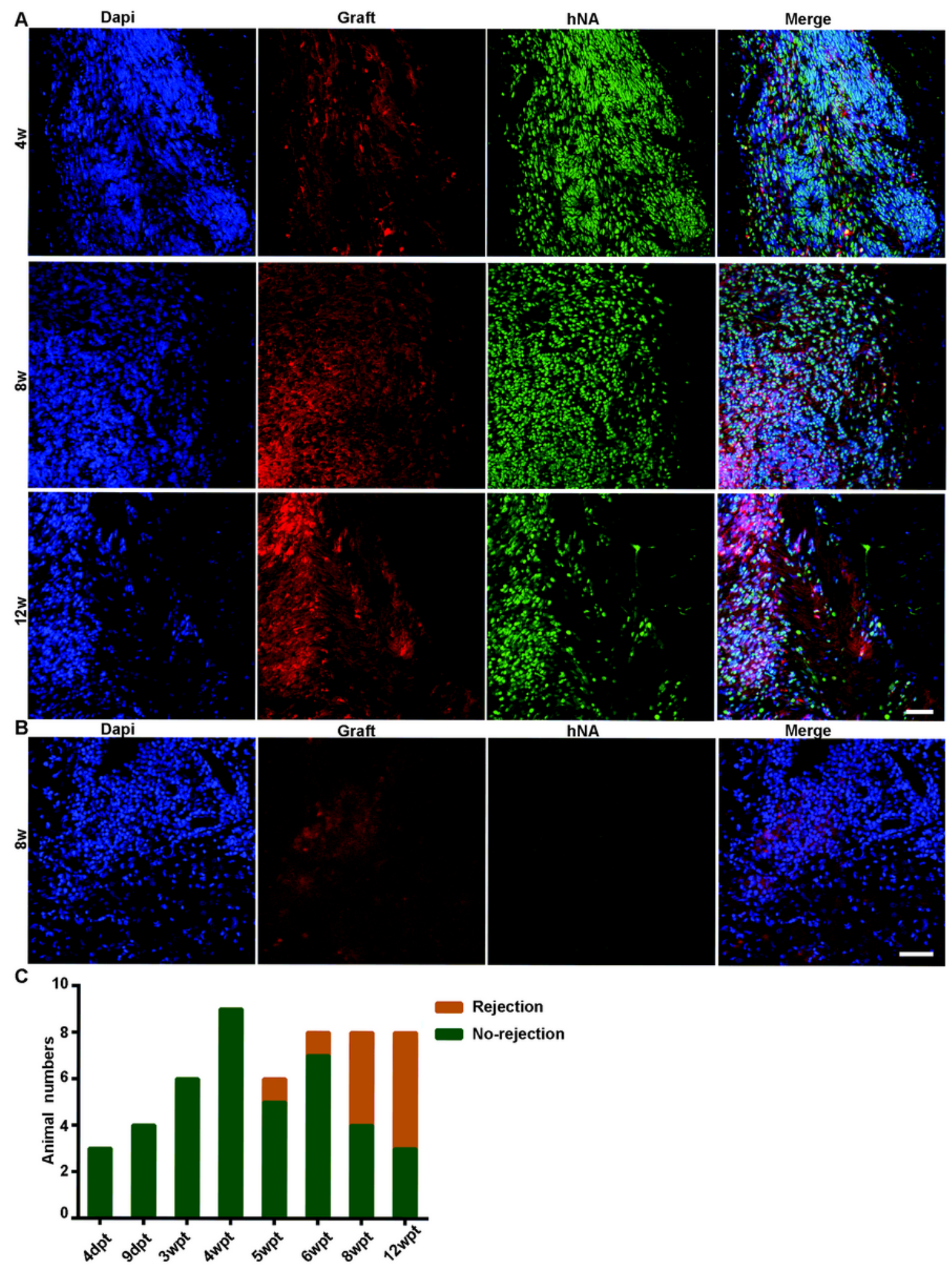

Figure 1

Early passaged human ESC-derived NPC grafts survived in immunocompetent adult rats up to 12 weeks post-transplantation (wpt). A, healthy human NPCs were confirmed by co-localization of Dsred fluorescence and human specific nuclear antigen, and demonstrated highly proliferation in vivo.Scale bar, $50 \mu \mathrm{m}$. B, a representative picture of massive human cell death occurred at late time points in some recipients, showed lost Dsred fluorescence and human specific nuclear antigen. C, population data 
showed that all human grafts survived healthy within $4 \mathrm{wpt}$, and a late-onset rejection occurred thereafter with a rejection rate more than $50 \%$ at 12 wpt for early passages.

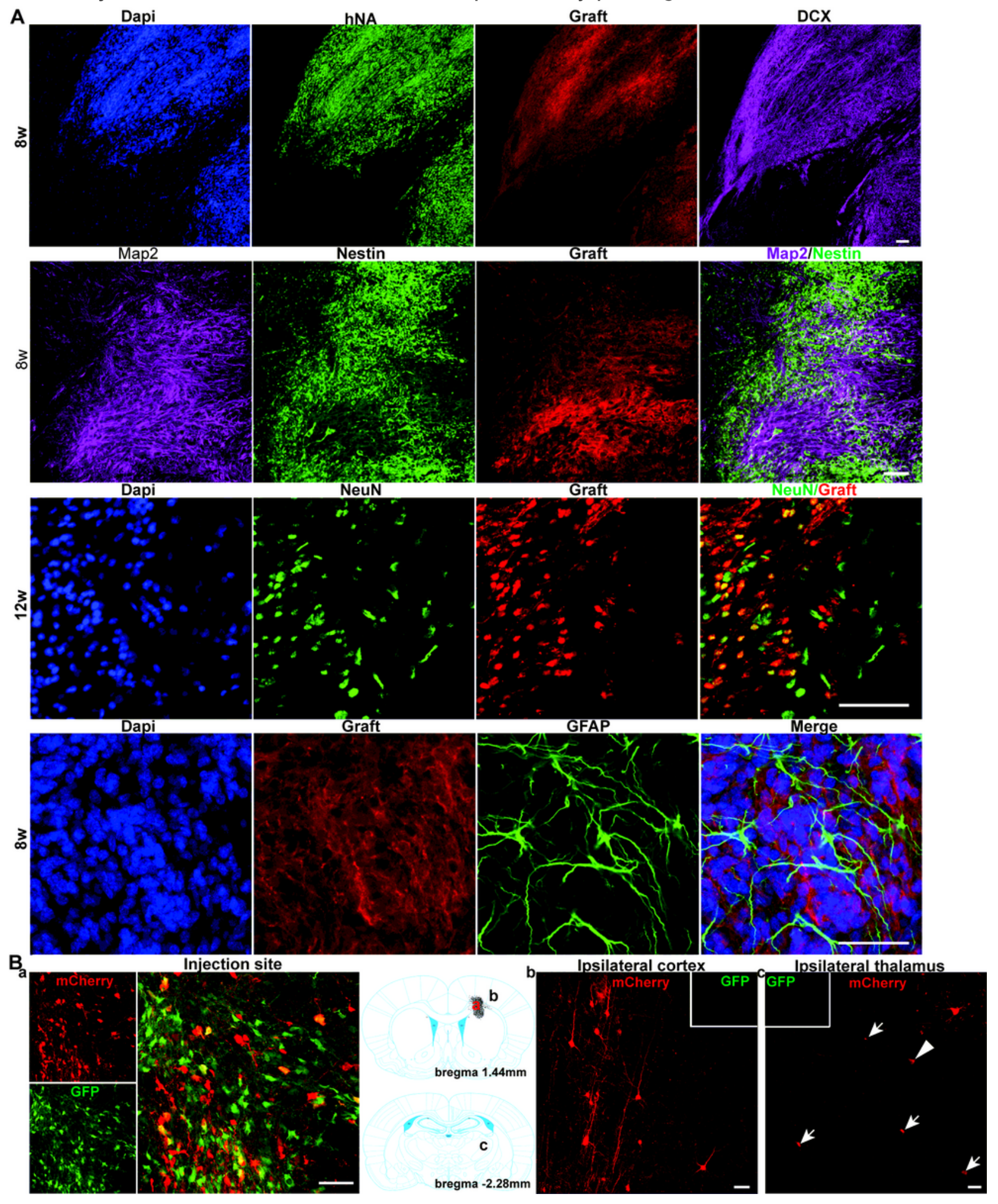

Figure 2

Engrafted human NPCs differentiated into neurons, and further incorporated into host neural network under settings without immunosuppression. A, grafted cells expressed pro-neuronal markers (DCx, MAP2 and NeuN), but not astrocyte marker (GFAP) within 12 weeks post-transplantation. B, retrograde 
monosynaptic tracing showed that long projection inputs from host neurons to engrafted cells originate from both ipsilateral neocortex and thalamus. B-a: the infected human cells co-expressed EGFP and mCherry fluorescence (as the starter neurons, EGFP+mCherry+); B-b,B-c: the traced host neurons only expressed mCherry (EGFP-(shown in inset), indicated by arrows and arrowhead) in the ipsilateral cortex (Bb) and the ipsilateral thalamus(B-C). The cell indicated with arrowhead was magnified in inset(upright corner). Scale bar, $50 \mu \mathrm{m}$.

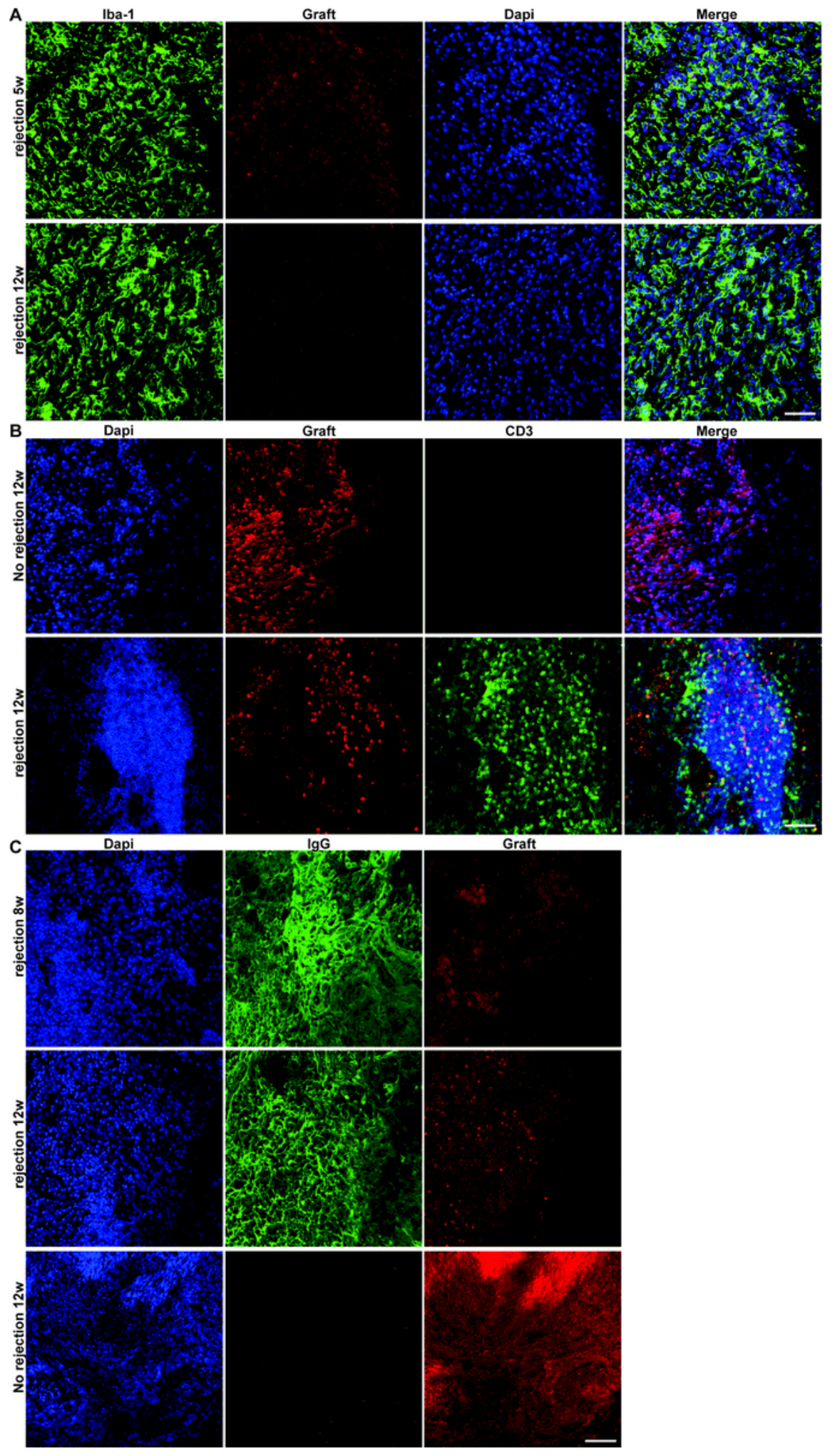

Figure 3 
The massive death of human NPC grafts was due to immune-rejection in the vast majority of cases. A, the graft area was reoccupied by the host cells with strong lba1 expression,Scale bar, $50 \mu \mathrm{m}$. B, the rejected grafts were infiltrated with $\mathrm{CD} 3+$ lymphocytes whereas non-rejected human grafts were completelydevoid of that,Scale bar, $50 \mu \mathrm{m}$. C, heavy host IgG deposited within rejected grafts but not in the non-rejecting brain,Scale bar, $50 \mu \mathrm{m}$.

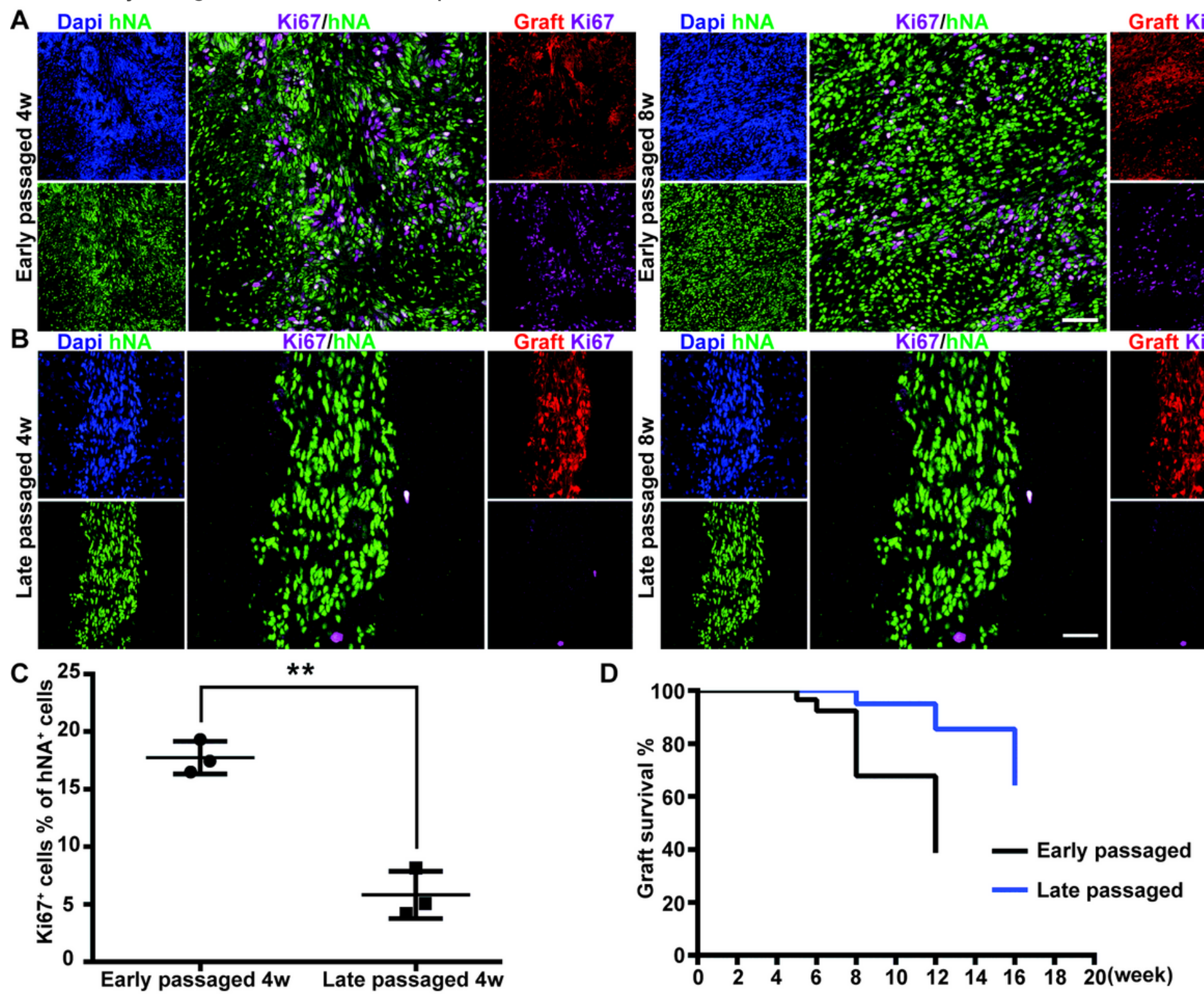

\section{Figure 4}

Late passaged human NPCs with low proliferation survived much longer than early passaged NPCs. A-B, early passaged NPCs showed relative high proliferation in vivo at 4-8 wpt, while late passaged NPCs showed very limited proliferation at 8 wpt. Scale bar, $50 \mu \mathrm{m}$. C, statistical analysis of proliferation levels of early and late NPCs at 4 wpt (unpaired T-test, $P<0.01^{\star \star}$ ). $\mathrm{N}=3$ biologically independent samples. $\mathrm{D}$, comparative analysis of graft surviving time of early and late passaged NPCs (Log-rank test, $P<0.01 \star \star)$. 


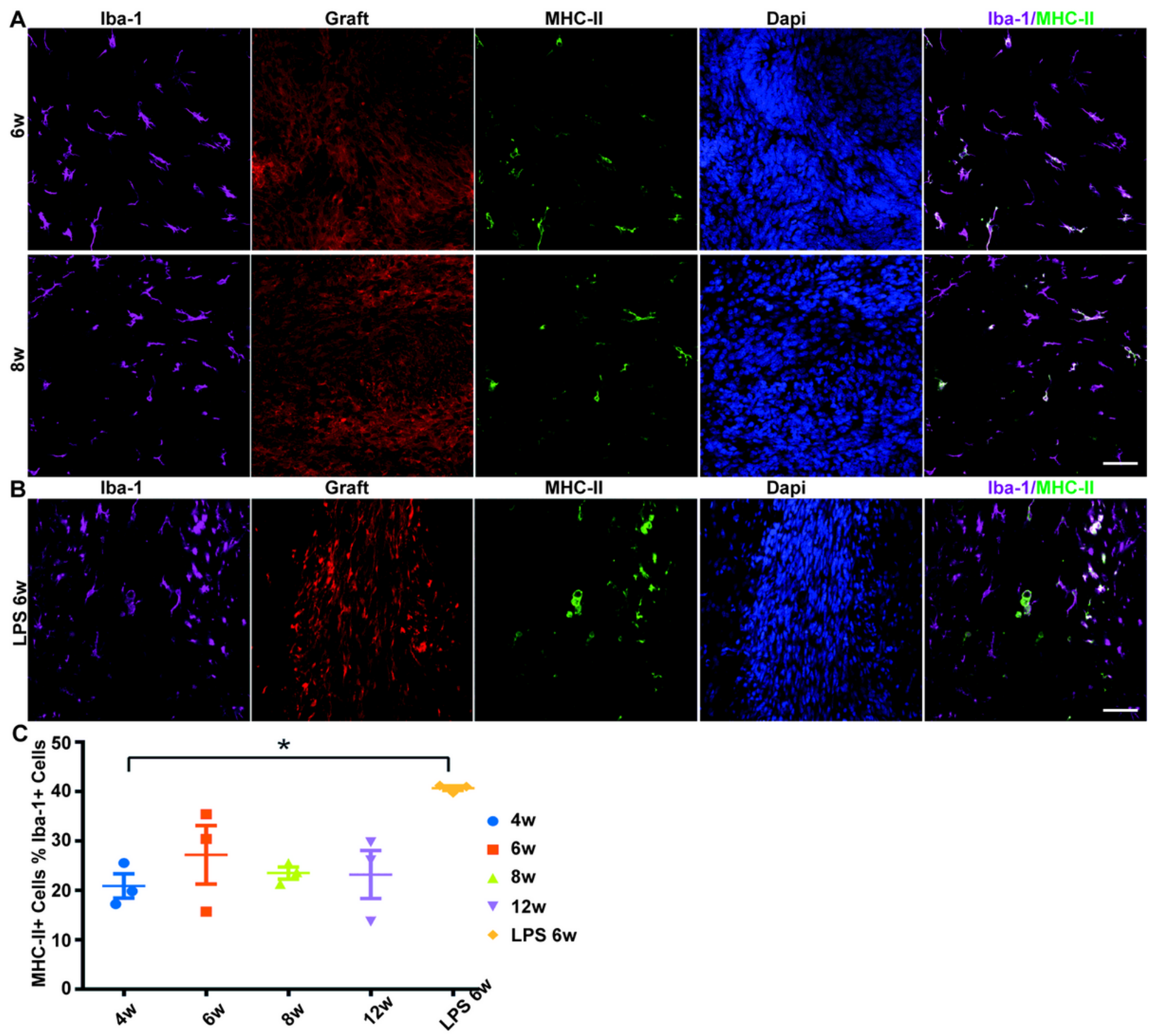

Figure 5

Representative pictures showed a subpopulation of microglia that upregulated MHC-II expression in response to the surviving human graft. $\mathrm{A}, \mathrm{MHC}$-II upregulation was specifically observed in microglia. $\mathrm{B}$, LPS challenge enhanced MHC-II expression.C, population data showed that the host MHC-II expression remained relatively low level with no changes over time (ANOVA, $P>0.05$ ). $N=3$ biologically independent samples. Scale bar, $50 \mu \mathrm{m}$.

\section{Supplementary Files}

This is a list of supplementary files associated with this preprint. Click to download. 
- sFigureandlegendsv1.pdf

Page 26/26 